\title{
An unusual polyp: a pedunculated leiomyoma of the sigmoid colon
}

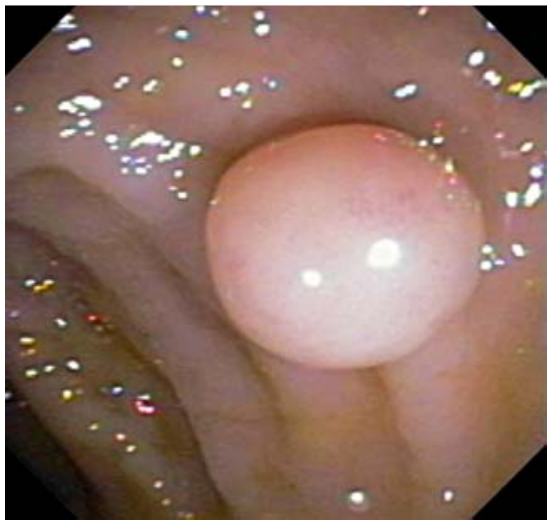

Fig. 1 Colonoscopic image demonstrating the pedunculated polyp in the sigmoid colon.

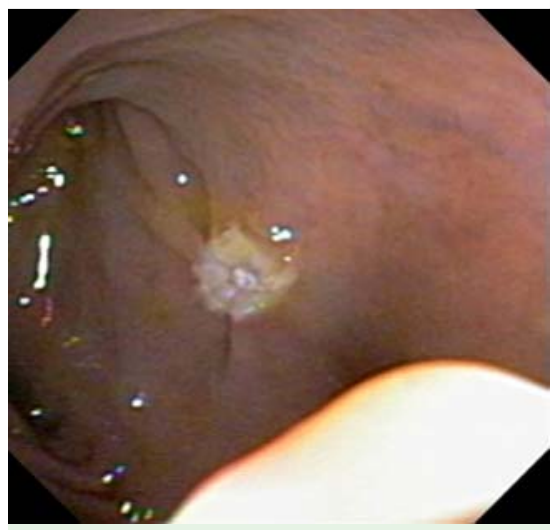

Fig. 2 Colonoscopic image following complete removal of the polyp by hot-snare polypectomy.

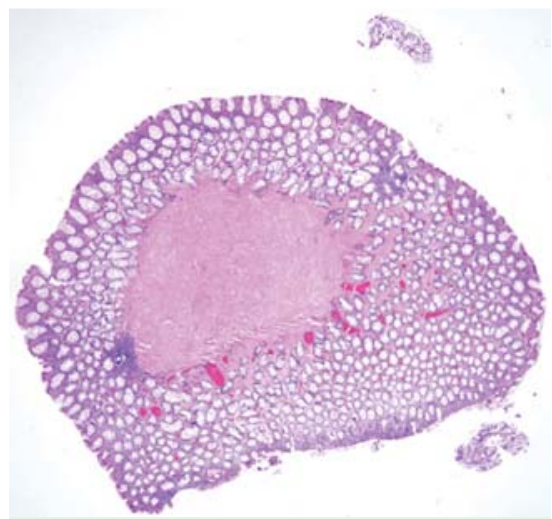

Fig. 3 Hematoxylin and eosin (H\&E)-stained section showing the leiomyomatous polyp.

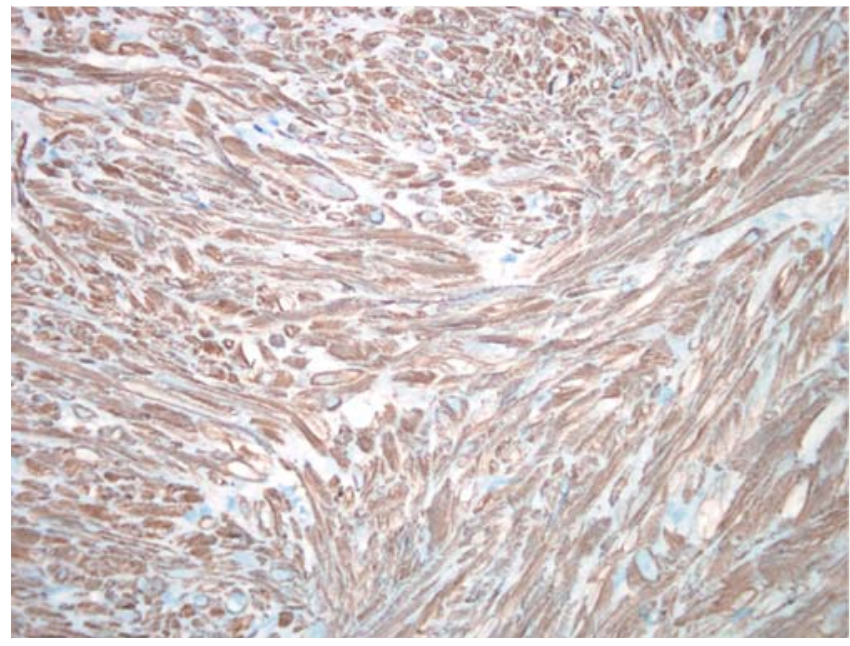

Fig. 4 Smooth muscle actin-stained section of the leiomyomatous polyp demonstrating the smooth muscle fibers.

A 56-year-old man presented for evaluation of scant rectal bleeding that occurred with bowel movements. He denied weight loss, fevers, fatigue, or alarm symptoms, and reported no family history of polyps, colorectal cancer, or intestinal disorders. His past medical history and physical examination including a digital rectal examination were unremarkable. Colonoscopy demonstrated a single $1.2 \mathrm{~cm}$ pedunculated polyp within the lumen of the sigmoid colon ( Fig. 1). The polyp, which was glistening, smooth, round, and on a minute stalk, was removed in its entirety by hot-snare polypectomy ( $\bullet$ Fig. 2).

Histologic sections of the polyp revealed normal mucosa overlying a well-circumscribed proliferation of bland, brightly eosinophilic spindle cells that were arising in association with the muscularis mucosae ( Fig. 3). On further staining, the neoplastic cells were positive for smooth muscle actin ( $\bullet$ Fig. 4) and negative for CD117 (not shown), consistent with a diagnosis of leiomyoma of the sigmoid colon.

Smooth muscle tumors, which include benign leiomyomas and malignant leiomyosarcomas, are the most common, nonepithelial tumors of the gastrointestinal tract [1]. The colon and rectum are rare sites for leiomyomas, representing $3 \%$ of all gastrointestinal leiomyomas [2]. The commonest location in the large bowel is the descending and sigmoid colon [3]. The peak incidence occurs in the third decade of life and there is a slight female pre- dominance [3]. Leiomyomas can present with a variety of symptoms including abdominal pain, constipation, weight loss, and bleeding [3].

The tumors arise from the muscularis mucosa or propria, or vascular smooth muscle [1]. On colonoscopy leiomyomas are intramural or pedunculated lesions that can be similar in appearance to adenomatous polyps [4]. Radiologic imaging such as computed tomography (CT) with colonography, barium enemas, and magnetic resonance imaging (MRI) can be useful diagnostic adjuncts [5].

Treatment includes complete removal, and recurrences are extremely rare. For small, pedunculated lesions, endoscopic snare polypectomy is a useful approach; for larger tumors or those where malignancy is a concern, surgical resection with wide margins is advocated [4].

\section{Endoscopy_UCTN_Code_CCL_1AD_2AC}

\section{Competing interests: None}

\section{D. Kemp ${ }^{1}$, C. A. Arnold ${ }^{2}$, M. S. Torben-} son $^{2}$, E. M. Stein ${ }^{3}$

1 Department of General Surgery, The Johns Hopkins Hospital, Baltimore, USA

2 Department of Pathology, The Johns Hopkins Hospital, Baltimore, USA

Division of Gastroenterology and Hepatology, Department of Medicine, The Johns Hopkins Hospital, Baltimore, USA 


\section{References}

1 Chow WH, Kwan WK, Ng WF. Endoscopic removal of leiomyoma of the colon. Hong Kong Med J 1997; 3: 325 - 327

2 Bjornsdottir H, Bjornsson J, Gudjonsson $H$. Leiomyomatous colonic polyp. Dig Dis Sci 1993; 38: 1945 - 1947

3 Hatch KF, Blanchard DK, Hatch GF et al. Tumors of the appendix and colon. World J Surg 2000; 24: 430-436
4 Jovanovic I, Cvejic T, Popovic D, Micev M. Endoscopic removal of pedunculated leiomyoma of the sigmoid colon (case report and literature review of diagnostic and treatment options). Acta Chir Iugosl 2006; 53: 87-89

5 Pickhardt PJ, Kim DH, Menias CO et al. Evaluation of submucosal lesions of the large intestine: part 1. Neoplasms. Radiographics 2007; 27: $1681-1692$
Bibliography

DOI $10.1055 / \mathrm{s}-0030-1256640$

Endoscopy 2011; 43: E306-E307

(c) Georg Thieme Verlag KG Stuttgart · New York . ISSN 0013-726X

\section{Corresponding author}

E. M. Stein, MD Assistant Professor of Medicine Division of Gastroenterology and Hepatology The Johns Hopkins Hospital

1830 E. Monument Street, Suite 429

Baltimore, MD 21205

USA

Fax: 1-410-614-7631

estein6@jhmi.edu 